\title{
GENERALIZED VECTOR VARIATIONAL INEQUALITIES WITH STAR-PSEUDOMONOTONE AND DISCONTINUOUS OPERATORS ${ }^{1}$
}

\author{
BUI TRONG KIEN ${ }^{2}$, NGAI CHING WONG ${ }^{3}$ AND JEN-CHIH YAO ${ }^{4}$
}

\footnotetext{
${ }^{1}$ This research was partially supported by grants from the National Science Council of Taiwan, ROC.

${ }^{2}$ Doctor, Department of Information and Technology, Hanoi University of Civil Engineering, 55 Giai Phong, Hanoi, Vietnam. Email:btkien@gmail.com

3,4 Professors, Department of Applied Mathematics, National Sun YatSen University, Kaohsiung, Taiwan. Email: wong@math.nsysu.edu.tw, yaojc@math.nsysu.edu.tw 
Abstract. In this paper we deal with the following generalized vector variational inequality problem: let $X, Y$ and $Z$ be topological vector spaces, $K$ be a convex set in $X, D$ be a nonempty set in $Y$, and $C$ be a closed convex cone in $Z$. Let $T: K \rightarrow 2^{D}$ be a multifunction and $f: K \times D \times K \rightarrow Z$ be a single-valued mapping. Find a point $\hat{x} \in K$ and $\hat{y} \in T(\hat{x})$ such that

$$
f(\hat{x}, \hat{y}, z) \notin-\operatorname{int} C, \quad \forall z \in K .
$$

We prove some existence theorems in which $T$ can be discontinuous, or $K$ can be unbounded, and a existence theorem in which $T$ is pseudomonotone.

Keywords. Generalized vector equilibrium problem, generalized vector variational inequality, upper semicontinuity, $C$-convex, $C$-upper semicontinuity, star-pseudomonotonicity.

2000 Mathematics Subject Classification: 49J30, 47H10, 47H17 


\section{INTRODUCTION}

Throughout this paper, let $Z$ be a (Hausdorff) topological vector space, and let $C$ be a closed and convex cone of $Z$ such that $\operatorname{int} C \neq \emptyset$ and $C \neq Z$. Here, int $C$ denotes the interior of $C$, while $A^{c}, \bar{A}$ and $\operatorname{co} A$ denotes the complement, the closure and the convex hull of a subset $A$ of $Z$, respectively.

A vector ordering in $Z$ is defined by setting

$$
z_{1} \leq z_{2} \text { if and only if } z_{2}-z_{1} \in C .
$$

Write

$$
z_{1}<z_{2} \text { if and only if } z_{2}-z_{1} \in \operatorname{int} C
$$

and

$$
z_{1} \nless z_{2} \text { if and only if } z_{2}-z_{1} \notin \operatorname{int} C \text {. }
$$

Let $X, Y$ be topological vector spaces, and let $K \subseteq X$ and $D \subseteq Y$ be nonempty. Let $T: K \rightarrow 2^{D}$ be a multifunction and $f: K \times \bar{D} \times$ $K \rightarrow Z$ be a single-valued mapping. The generalized vector variational inequality $\operatorname{GVVI}(K, T, f)$ is the following problem:

(V) Find $\hat{x} \in K$ such that

$$
\exists \hat{y} \in T(\hat{x}), \forall z \in K \text {, we have } f(\hat{x}, \hat{y}, z) \nless 0 .
$$

Together with model $(\mathrm{V})$ of $\operatorname{GVVI}(K, T, f)$, the following auxiliary models is also of interest:

(W) Find $\hat{x} \in K$ such that

$$
\forall z \in K, \exists \hat{y} \in T(\hat{x}) \text {, we have } f(\hat{x}, \hat{y}, z) \nless 0 \text {, }
$$

and the so-called Minty vector variational inequality:

(M) Find $\hat{x} \in K$ such that

$$
\forall z \in K, \forall \hat{y} \in T(z) \text {, we have } f(\hat{x}, \hat{y}, z) \nless 0 \text {. }
$$

We will denote by $S_{V}, S_{W}$ and $S_{M}$ the solution set of problems $(V)$, $(W)$ and $(M)$, respectively. A point $\hat{x} \in S_{V}$ is called a strong solution of $\operatorname{GVVI}(K, T, f)$, while a point $x_{0}$ in $S_{W}$ is called a weak solution.

It is interesting that problem $(V)$ covers several generalized variational inequalities and equilibrium problems. Here are some examples.

(GVI) Put $Z=\mathbb{R}, C=\mathbb{R}_{+}, Y=X^{*}=D$ and $f(x, y, z)=\langle y, z-x\rangle$. Then $(V)$ reduces to the generalized variational inequality problem of finding:

$$
\hat{x} \in K \text { and } \hat{y} \in T(\hat{x}) \text { such that }\langle\hat{y}, z-\hat{x}\rangle \geq 0, \forall z \in K .
$$


(EP) Put $Z=\mathbb{R}, C=\mathbb{R}_{+}, Y=X=D$ and $f(x, y, z)=g(x, z)+$ $h(z)-h(x)$ where $g$ is a real valued function on $K \times K$ such that $g(x, x)=0$ for all $x \in K$. Then $(V)$ reduces to the equilibrium problem of finding:

$$
\hat{x} \in K \text { such that } g(\hat{x}, z)+h(z)-h(\hat{x}) \geq 0, \forall z \in K .
$$

(VI) Put $Z=\mathbb{R}, C=\mathbb{R}_{+}, Y=D$ and $f(x, y, z)=\phi(y, z)-\phi(y, x)$ then $(V)$ reduces to the general variational inequality problem of finding:

(3) $\hat{x} \in K$ and $\hat{y} \in T(\hat{x})$ such that $\phi(\hat{y}, z)-\phi(\hat{y}, \hat{x}) \geq 0, \forall z \in K$.

Problem (VI) has been studied recently by Aussel and Luc (2005).

The existence of solutions of (GVI) has been studied by many authors. See for instance Cubiotti (1997-2005), Li (2004), Yao (19921994), Yen (1994, 1995), and references therein. Also, results about the existence of weak solutions for generalized vector variational inequality problem were investigated by many authors. See for instance Ansari (1999), Chen and Craven (1990), Lee et al. (1997). Very few papers appeared in the literature discuss about the existence of strong solution of $\operatorname{GVVI}(K, T, f)$, however.

The aim of this paper is to derive some existence results of strong solutions for $\operatorname{GVVI}(K, T, f)$ with discontinuous operators and starpseudomonotone operators. Namely, we will prove some existence theorems in which $T$ can be discontinuous and $K$ can be unbounded. Our results extends some previous results. Beside, an existence theorem involving star-pseudomonotne operators is also established. In order to obtain these results, we utilize the fixed point theorem due to Ky Fan and the scalarization method.

In Section 2 we recall some auxiliary results. Section 3 is destined for presenting existence results in the case discontinuous operators are involved. Section 4 will be devoted to a existence result for the case where the operators are star-pseudomonotone.

\section{Preliminaries}

Here are the basic setting in this paper. Let $X$ and $Y$ be topological vector spaces, and let $Z$ be a locally convex space. Let $C$ be a closed and convex proper cone in $Z$ with nonempty interior. Let $K$ be a nonempty convex subset of $X$, and $D$ be a nonempty convex subset of $Y$. Let $T: K \rightarrow 2^{D}$ be a multifunction. We denote by $\Gamma_{T}$ the graph of $T$, that is, the set $\{(x, y) \in X \times Y \mid y \in T(x)\}$.

Definition 2.1. (a) $T$ is said to be lower semicontinuous (l.s.c., shortly) at $\bar{x} \in K$ if for any open set $V$ in $E$ such that $V \cap T(\bar{x}) \neq \emptyset$, 
there exists a neighborhood $U$ of $\bar{x}$ such that

$$
V \cap T(x) \neq \emptyset, \quad \forall x \in U \cap K .
$$

(b) $T$ is said to be upper semicontinuous (u.s.c., shortly) at $\bar{x} \in K$ if for any open set $V$ in $E$ such that $T(\bar{x}) \subset V$, there exists a neighborhood $W$ of $\bar{x}$ with the property that

$$
T(x) \subset V, \quad \forall x \in W \cap K .
$$

(c) $T$ is said to be lower semicontinuous (resp., upper semicontinuous) on $K$ if it is l.s.c. (resp., u.s.c.) at every point $x \in K$.

(d) A single-valued mapping $g: X \rightarrow Z$ is said to be $C$-upper semicontinuous on $X$ if for every $z \in Z$ the set $g^{-1}(z-\operatorname{int} C)$ is open in $\mathrm{X}$.

Tanaka (1997) proved that $g$ is $C$ - upper semicontinuous on $X$ if and only if for each fixed $x \in X$ and for any $y \in \operatorname{int} C$, there exists a neighborhood $U$ of $x$ such that

$$
g(u) \in g(x)+y-\operatorname{int} C, \quad \forall u \in U .
$$

(e) $g$ is $C$-lower semicontinuous on $X$ if for each fixed $x \in X$ and for any $y \in \operatorname{int} C$, there exists a neighborhood $U$ of $x$ such that

$$
g(x) \in g(u)+y-\operatorname{int} C, \quad \forall u \in U .
$$

(f) If $g$ is $C$-lower semicontinuous and $C$-upper semicontinuous on $X$ then $g$ is called $C$ - continuous on $X$.

(g) A single-valued mapping $h: K \rightarrow Z$ is called $C$-convex if for every $x, x^{\prime} \in K$ and $t \in[0,1]$ one has

$$
t h(x)+(1-t) h\left(x^{\prime}\right)-h\left(t x+(1-t) x^{\prime}\right) \in C .
$$

It is called $C$-strongly convex if for every $x, x^{\prime} \in K$ and $t \in[0,1]$ one has

$$
\operatorname{th}(x)+(1-t) h\left(x^{\prime}\right)-h\left(t x+(1-t) x^{\prime}\right) \in \operatorname{int} C \cup\{0\} .
$$

If $-h$ is $C$-convex (resp., $C$-strongly convex) then $h$ is said to be $C$-concave (resp., $C$-strongly concave) on $K$.

We continue by recalling the scalarization method. Let $Z^{*}$ be the topological dual of $Z$. The set

$$
C^{*}:=\left\{z^{*} \in Z^{*}:\left\langle z^{*}, z\right\rangle \geq 0 \text { for all } z \in C\right\}
$$

is the polar cone of $C$. Note that $C^{*}$ has a weakly-star compact base $B$, that is, $C^{*}=\bigcup_{t>0} t B$, and $B$ is convex and weakly-star compact with $0 \notin B$ (see Luc, 1989). When $\operatorname{int} C \neq \emptyset$ and $\bar{z} \in \operatorname{int} C, \bar{z} \neq 0$, the set

$$
B=\left\{z^{*} \in C^{*}:\left\langle z^{*}, \bar{z}\right\rangle=1\right\}
$$

is a weakly- star compact convex base for $C^{*}$. We put $C_{+}^{*}=C^{*} \backslash\{0\}$. 
Note that from the bipolar theorem we have

(4) $z \in C \Longleftrightarrow\left[\left\langle z^{*}, z\right\rangle \geq 0, \forall z^{*} \in C^{*}\right] \Longleftrightarrow\left[\left\langle z^{*}, z\right\rangle \geq 0, \forall z^{*} \in B\right]$

(5) $z \in \operatorname{int} C \Longleftrightarrow\left[\left\langle z^{*}, z\right\rangle>0, \forall z^{*} \in C_{+}^{*}\right] \Longleftrightarrow\left[\left\langle z^{*}, z\right\rangle>0, \forall z^{*} \in B\right]$.

(see Jeyakumar et al., (1993) for developments).

The following proposition is a useful tool for the scalarization method.

Proposition 2.2. Let $g$ be a single-valued mapping from $K$ into $Z$ and $u^{*} \in C_{+}^{*}$. Let $\phi: K \rightarrow \mathbb{R}$ be a mapping defined by $\phi(x)=\left\langle u^{*}, g(x)\right\rangle$ for all $x \in K$. Then the following assertions are valid:

(a) If $g$ is $C$-convex (resp., $C$-concave) then $\phi$ is convex (resp., concave);

(b) If $g$ is $C$-upper semicontinuous (resp., $C$-lower semicontinuous) then $\phi$ u.s.c. (resp., l.s.c.)

Proof. (a) Since $g$ is $C$-convex, then for all $x, x^{\prime} \in K$ and $t \in[0,1]$ one has

$$
\operatorname{tg}(x)+(1-t) g\left(x^{\prime}\right)-g\left(t x+(1-t) x^{\prime}\right) \in C .
$$

By (5) we have $\left\langle u^{*}, t g(x)+(1-t) g\left(x^{\prime}\right)-g\left(t x+(1-t) x^{\prime}\right)\right\rangle \geq 0$. Hence

$$
t\left\langle u^{*}, g(x)\right\rangle+(1-t)\left\langle u^{*}, g\left(x^{\prime}\right)\right\rangle \geq\left\langle u^{*} g\left(t x+(1-t) x^{\prime}\right)\right\rangle .
$$

This implies that

$$
t \phi(x)+(1-t) \phi\left(x^{\prime}\right) \geq \phi\left(t x+(1-t) x^{\prime}\right)
$$

and so $\phi$ is convex. By a similar argument we show that if $g$ is $C$-concave then $\phi$ is concave.

(b) Let $x_{n} \rightarrow x$. We want to show that $\limsup _{n \rightarrow \infty} \phi\left(x_{n}\right) \leq \phi(x)$. Choose $y_{j} \in \operatorname{int} C$ such that $y_{j} \rightarrow 0$. Then for each $j>0$ there exists a neighborhood $U_{j}$ of $x$ such that

$$
g(u) \in g(x)+y_{j}-\operatorname{int} C, \quad \forall u \in U_{j} .
$$

Therefore for each $j$ there exists $n_{j}>0$ such that

$$
g\left(x_{n}\right) \in g(x)+y_{j}-\operatorname{int} C, \quad \forall n>n_{j} .
$$

By (5) it follows that $\left\langle u^{*}, g\left(x_{n}\right)-g(x)-y_{j}\right\rangle<0$. Hence

$$
\begin{aligned}
\phi\left(x_{n}\right) & =\left\langle u^{*}, g\left(x_{n}\right)-g(x)-y_{j}+g(x)+y_{j}\right\rangle \\
& =\left\langle u^{*}, g\left(x_{n}\right)-g(x)-y_{j}\right\rangle+\left\langle u^{*}, g(x)+y_{j}\right\rangle \\
& <\left\langle u^{*}, g(x)\right\rangle+\left\langle u^{*}, y_{j}\right\rangle
\end{aligned}
$$

for all $n>n_{j}$. This implies that

$$
\limsup _{n \rightarrow \infty} \phi\left(x_{n}\right) \leq\left\langle u^{*}, g(x)\right\rangle+\left\langle u^{*} y_{j}\right\rangle \text {. }
$$


Letting $j \rightarrow \infty$ and noting that $\left\langle u^{*}, y_{j}\right\rangle \rightarrow 0$, we obtain

$$
\limsup _{n \rightarrow \infty} \phi\left(x_{n}\right) \leq\left\langle u^{*}, g(x)\right\rangle=\phi(x) \text {. }
$$

By the same way, we obtain the second assertion of $(b)$.

One of the tools in deriving our results is Ky Fan theorem. We state it below for the convenience.

Theorem 2.3 (Ky Fan). Let $K$ be a nonempty subset of a topological vector space $X$ and $G: K \rightarrow 2^{X}$ be a multifunction with closed values such that the convex hull of every finite subset $\left\{x_{1}, x_{2}, \ldots, x_{n}\right\}$ of $X$ is contained in the corresponding union $\bigcup_{i=1}^{n} G\left(x_{i}\right)$. If there exists $x_{0} \in K$ such that $G\left(x_{0}\right)$ is compact, then $\bigcap_{x \in K} G(x) \neq \emptyset$.

We recall that the multifunction $G$ satisfying the property in Theorem 2.3 is called a KKM mapping.

We also need the following Sion's minimax theorem (see Jeyakumar et al., 1993).

Theorem 2.4 (Sion). Let $A$ and $B$ be convex subset of some real topological vector spaces with $B$ compact, and let $p: A \times B \rightarrow \mathbb{R}$. If $p(., b)$ is lower semicontinuous and quasiconvex on $A$ for all $b \in B$, and if $p(a,$.$) is upper semicontinuous and quasiconcave on B$ for all $a \in A$, then

$$
\inf _{a \in A} \max _{b \in B} p(a, b)=\max _{b \in B} \inf _{a \in A} p(a, b) .
$$

\section{Existence of SOLUTiOns in CASE OF Discontinuous OPERATORS}

In addition to the basic assumptions stated in the beginning of Section 2, we further assume in this section that $f: K \times D \times K \rightarrow Z$ is a single-valued mapping satisfying some of the following conditions

(i) the set $T(x)$ is nonempty, weakly compact, and convex for all $x \in K$

(ii) there exists $u^{*} \in C_{+}^{*}$ such that for each $z \in K$, the set

$$
F(z)=\left\{x \in K \mid \sup _{y \in T(x)}\left\langle u^{*}, f(x, y, z)\right\rangle \geq 0\right\}
$$

is closed;

(iii) for each $x \in K$ and $y \in T(x)$ one has $f(x, y, x)=0$;

(iv) for each $x \in K$ and $y \in T(x)$, the function $f(x, y, \cdot)$ is $C$-convex and $C-$ l.s.c. on $K$;

(v) for each $(x, z) \in K \times K$, the function $f(x, \cdot, z)$ is $C$-concave and $C-$ u.s.c. on $D$. 
Theorem 3.1. Let $X$ be a dual Banach space and $Y$ be a locally convex space. Assume conditions (i), (ii), (iii), (vi), and (v) hold. Assume further that $F(z)$ is weakly* closed for all $z$ in $K$, and

(vi) There is a bounded subset $K_{0}$ of $K$ such that for each $x \in K \backslash K_{0}$ there exists $z \in K_{0}$ with $f(x, y, z)<0$ for all $y \in T(x)$.

Then there exists a point $\hat{x} \in K_{0}$ such that

$$
\max _{y \in T(\hat{x})}\left\langle u^{*}, f(\hat{x}, y, z)\right\rangle \geq 0, \quad \forall z \in K .
$$

Moreover, there exists $\hat{y} \in T(\hat{x})$ such that

$$
f(\hat{x}, \hat{y}, z) \nless 0, \quad \forall z \in K .
$$

Proof. We choose $r>0$ such that int $B_{r}$ contains $K_{0}$, where $B_{r}=\{x \in$ $X:\|x\| \leq r\}$ is the closed ball in $X$ with radius $r$. Set $\Omega=K \cap B_{r}$. We define a multifunction $G: \Omega \rightarrow 2^{X}$ by the formula

$$
G(z)=\left\{x \in \Omega \mid \sup _{y \in T(x)}\left\langle u^{*}, f(x, y, z)\right\rangle \geq 0\right\} \text { for } z \in \Omega .
$$

By Proposition 2.2, the function $y \mapsto\left\langle u^{*}, f(x, y, z)\right\rangle$ is concave and u.s.c. In particular, for each real number $\delta$, the convex set $\{y \in Y$ : $\left.\left\langle u^{*}, f(x, y, z)\right\rangle \geq \delta\right\}$ is closed, and thus weakly closed, in the locally convex space $Y$. Hence, this function is also weakly u.s.c. Since $T(x)$ is a weakly compact set, we have

$$
G(z)=\left\{x \in \Omega \mid \max _{y \in T(x)}\left\langle u^{*}, f(x, y, z)\right\rangle \geq 0\right\} .
$$

We claim that $G$ is a KKM mapping. Indeed, by (iii) we have $z \in$ $G(z)$ for all $z \in \Omega$. Hence $G(z) \neq \emptyset$ for all $z \in \Omega$. Since

$$
G(z)=F(z) \cap B_{r},
$$

$G(z)$ is a weak* compact subset of $\Omega$, by Banach-Alaoglu's Theorem. Taking any $z_{1}, z_{2} \in \Omega$ we show that $\operatorname{co}\left\{z_{1}, z_{2}\right\} \subset G\left(z_{1}\right) \cup G\left(z_{2}\right)$. Let $z \in \operatorname{co}\left\{z_{1}, z_{2}\right\}$. Then $z=t z_{1}+(1-t) z_{2}$ for some $t \in[0,1]$. When $t=0$ or $t=1$, the claim is trivial. Consider $t \in(0,1)$, with $z \notin G\left(z_{i}\right) ; i=$ 1,2 . Since $z \in \Omega$, we obtain

$$
\max _{y \in T(z)}\left\langle u^{*}, f\left(z, y, z_{i}\right)\right\rangle<0 ; i=1,2 .
$$

By (iv) and Proposition 2.2, the function $\left\langle u^{*}, f(x, y, \cdot)\right\rangle$ is convex. By (iii) we have

$$
\begin{aligned}
0 & =\max _{y \in T(z)}\left\langle u^{*}, f(z, y, z)\right\rangle \\
& \leq t \max _{y \in T(z)}\left\langle u^{*}, f\left(z, y, z_{1}\right)\right\rangle+(1-t) \max _{y \in T(z)}\left\langle u^{*}, f\left(z, y, z_{2}\right)\right\rangle, \\
& <0,
\end{aligned}
$$


which is an absurd. Thus we must have $z \in G\left(z_{1}\right) \cup G\left(z_{2}\right)$ and so $\operatorname{co}\left\{z_{1}, z_{2}\right\} \subset G\left(z_{1}\right) \cup G\left(z_{2}\right)$. By a similar argument we can show that

$$
\operatorname{co}\left\{z_{1}, z_{2}, \ldots, z_{n}\right\} \subset \bigcup_{i=1}^{n} G\left(z_{i}\right)
$$

for any finite subset $\left\{x_{1}, x_{2}, \ldots, x_{n}\right\}$ in $\Omega$.

By Theorem 2.3,

$$
\exists \hat{x} \in \Omega \text { such that } \hat{x} \in \bigcap_{z \in \Omega} G(z) .
$$

In other words,

$$
\exists \hat{x} \in \Omega, \max _{y \in T(\hat{x})}\left\langle u^{*}, f(\hat{x}, y, z)\right\rangle \geq 0, \quad \forall z \in \Omega .
$$

By (vi), we see that $\hat{x} \in K_{0}$.

We need to show that

$$
\max _{y \in T(\hat{x})}\left\langle u^{*}, f(\hat{x}, y, z)\right\rangle \geq 0, \quad \forall z \in K .
$$

Take any $z \in K$. Since $\hat{x} \in \operatorname{int} B_{r}$ we have $\hat{x}+t(z-\hat{x}) \in K \cap \operatorname{int} B_{r}$ for a sufficiently small $t \in(0,1)$. Then $(7)$ implies that

$$
\begin{aligned}
& \max _{y \in T(\hat{x})}\left\langle u^{*}, f(\hat{x}, y, \hat{x}+t(z-\hat{x})\rangle\right. \\
= & \max _{y \in T(\hat{x})}\left\langle u^{*}, f(\hat{x}, y,(1-t) \hat{x}+t z)\right\rangle \geq 0 .
\end{aligned}
$$

By the convexity of the function $\left\langle u^{*}, f(x, y, \cdot)\right\rangle$, we obtain from $(9)$ the following:

$$
\begin{aligned}
& 0 \leq \max _{y \in T(\hat{x})}\left\langle u^{*}, f(\hat{x}, y,(1-t) \hat{x}+t z)\right\rangle \\
& \leq t \max _{y \in T(\hat{x})}\left\langle u^{*}, f(\hat{x}, y, z)\right\rangle+(1-t) \max _{y \in T(\hat{x})}\left\langle u^{*}, f(\hat{x}, y, \hat{x})\right\rangle
\end{aligned}
$$

Since $f(\hat{x}, y, \hat{x})=0$, it follows $(8)$.

To complete the proof we will use the Sion's theorem (Theorem 2.4). Put $p(z, y)=\left\langle u^{*}, f(\hat{x}, y, z)\right\rangle$. By the assumptions of the theorem and Proposition 2.2, the function $p(\cdot, y)$ is l.s.c. and convex. Also, the function $p(z, \cdot)$ is u.s.c. and concave, and thus weakly u.s.c. By Theorem 2.4 and (8) we get

$$
0 \leq \inf _{z \in K} \max _{y \in T(\hat{x})} p(z, y)=\max _{y \in T(\hat{x})} \inf _{z \in K} p(z, y) .
$$

Since $y \mapsto p(z, y)$ is weakly u.s.c., the function $y \mapsto \inf _{z \in K} p(z, y)$ is also weakly u.s.c. Hence, there exists $\hat{y} \in T(\hat{x})$ such that

$$
\inf _{z \in K} p(z, \hat{y})=\max _{y \in T(\hat{x})} \inf _{z \in K} p(z, y) \geq 0 .
$$


It follows that $\left\langle u^{*}, f(\hat{x}, \hat{y}, z)\right\rangle \geq 0, \forall z \in K$. This is equivalent to $\left\langle u^{*},-f(\hat{x}, \hat{y}, z)\right\rangle \leq 0, \forall z \in K$. By $(5)$ we have $-f(\hat{x}, \hat{y}, z) \notin \operatorname{int} C$ for all $z \in K$, and (6) is obtained. The proof of the theorem is complete.

In Theorem 3.1, condition (ii) is checked for a particular element $u^{*} \in$ $C_{+}^{*}$. It seems that we do not know when such $u^{*}$ exists. Fortunately, under certain conditions, for an example, $T$ is upper semicontinuous and $f(\cdot, \cdot, z)$ is $C$-continuous, we can choose any $u^{*} \in C_{+}^{*}$.

The following example illustrates our result.

Example 3.2. Let $X=\mathbb{R}, K=[0,1] \subset X, Y=\mathbb{R}, D=[1,4], Z=\mathbb{R}^{2}$ and

$$
C=\mathbb{R}_{+}^{2}=\{(x, y) \mid x \geq 0, y \geq 0\} .
$$

Let $T$ and $f$ be defined by

$$
T(x)= \begin{cases}{[2,4],} & \text { if } x=0 \\ \{1\}, & \text { if } 0<x \leq 1\end{cases}
$$

and

$$
f(x, y, z)=\left(f_{1}(x, y, z), f_{2}(x, y, z)\right)
$$

where

$$
f_{1}(x, y, z)=y\left(z^{2}-x^{2}\right) \text { and } f_{2}(x, y, z)=y(z-x) .
$$

Then the set $\{0\} \times[2,4]$ is a solution set of $\operatorname{GVVI}(K, T, f)$. Moreover, $T$ is not upper semicontinuous on $[0,1]$.

Proof. First we note that

$$
C^{*}=\{(u, v) \mid u \geq 0, v \geq 0\} ;-\operatorname{int} C=\{(x, y) \mid x<0, y<0\} .
$$

Taking $u^{*}=(1,0)$ we want to check all conditions of Theorem 3.1.

(i) It is obvious that $T(x)$ is a nonempty compact convex set for each $x \in[0,1]$.

(ii) For each $z \in[0,1]$ we have

$$
\begin{aligned}
& \left\{x \in K \mid \sup _{y \in T(x)}\left\langle u^{*}, f(x, y, z)\right\rangle \geq 0\right\} \\
= & \left\{x \in[0,1] \mid \sup _{y \in T(x)} y\left(z^{2}-x^{2}\right) \geq 0\right\} \\
= & {[0, z] . }
\end{aligned}
$$

This is a closed set.

(iii) For each $x \in M$ and $y \in T(x)$ we have $f(x, y, x)=(0,0)$.

(iv) For each $x \in K$ and $y \in T(x) \subset[1,4]$, the functions $f_{1}(x, y, \cdot)$ and $f_{2}(x, y, \cdot)$ are convex and continuous. Hence, $f$ is $C$-convex and $C$-lower semicontinuous. 
(v) For each $x, z \in K \times K$ the functions $f_{1}(x, ., z)$ and $f_{2}(x, \cdot, z)$ are linear and continuous.

(vi) is plain, as $K$ is compact.

Thus all conditions of Theorem 3.1 are fulfilled. Taking $\hat{x}=0$ and $\hat{y} \in T(0)=[2,4]$, we have

$$
f(0, \hat{y}, z)=\left(y z^{2}, y z^{2}\right) \nless 0, \forall z \in[0,1] .
$$

Hence the set $\{0\} \times[2,4]$ is a solution set of the problem.

It is observed that $T$ is not upper semicontinuous on $K$. Indeed, $x_{n}=1 / n \rightarrow 0, y_{n}=1 \in T\left(x_{n}\right)$ but $1 \notin T(0)=[2,4]$.

Theorem 3.1 requires that $X$ is a dual Banach space. This requirement guarantees the weak* compactness of weakly* closed bounded sets. The following results relax this assumption by strengthening the coercive condition (vi).

Theorem 3.3. Let $Y$ be a locally convex space (resp. topological vector space). In addition to conditions (i), (ii), (iii), (iv) and (v), suppose further that $T(x)$ is weakly compact (resp. compact) each $x$ in $K, F(z)$ is weakly closed (resp. closed) for each $z$ in $K$, and

(vi)' there exists a weakly compact (resp. compact) $K_{0}$ of $K$ and $z_{0} \in$ $K$ such that for each $x \in K \backslash K_{0}$ one has $f\left(x, y, z_{0}\right)<0$ for all $y \in T(x)$.

Then there exists a point $\hat{x} \in K$ and $\hat{y} \in T(\hat{x})$ such that

$$
f(\hat{x}, \hat{y}, z) \nless 0, \quad \forall z \in K .
$$

Proof. Assume that $Y$ is locally convex. By condition (v),

$$
F(z)=\left\{x \in K \mid \max _{y \in T(x)}\left\langle u^{*}, f(x, y, z)\right\rangle \geq 0\right\} .
$$

We claim that $F\left(z_{0}\right) \subset K_{0}$. Indeed, suppose that the claim is false. Then there is an $x \in F\left(z_{0}\right) \backslash K_{0}$ and so $x \in K \backslash K_{0}$. By (vi), $-f\left(x, y, z_{0}\right) \in \operatorname{int} C$ for all $y \in T(x)$. By (5), it follows that $\left\langle u^{*},-f\left(x, y, z_{0}\right)\right\rangle>0$ for all $y \in T(x)$. Hence

$$
\max _{y \in T(x)}\left\langle u^{*}, f\left(x, y, z_{0}\right)\right\rangle<0 .
$$

This implies that $x \notin F\left(z_{0}\right)$, which is absurd. Thus $F\left(z_{0}\right)$ is a weakly compact set. As in the proof of Theorem 3.1, we can show that $F(z)$ is a KKM mapping, and the desired conclusion follows accordingly. The case $Y$ is not locally convex is similar.

Theorem 3.4. Let $X$ and $Y$ be a topological vector spaces. Assume conditions (i), (ii), (iii), (iv) and (v) hold. Suppose further that 
(vi)" there exist compact sets $K_{1} \subseteq K_{2}$ in $K$ such that $K_{1}$ is finitedimensional, and for each $x \in K \backslash K_{2}$ there exists $z \in K_{1}$ with $f(x, y, z)<0$ for all $y \in T(x)$.

Then there exists a point $\hat{x} \in K_{2}$ and $\hat{y} \in T(\hat{x})$ such that

$$
f(\hat{x}, \hat{y}, z) \nless 0, \quad \forall z \in K .
$$

Proof. We denote by $\mathcal{F}$ the set of all finite-dimensional subspaces of $X$ containing $K_{1}$. Fix any $S \in \mathcal{F}$ and put

$$
\Omega=K \cap S, \quad T^{S}=\left.T\right|_{\Omega}, \quad f^{S}=\left.f\right|_{\Omega \times D \times \Omega} .
$$

The task is to check that Theorem 3.1 can be applied to the problem $\operatorname{GVVI}\left(\Omega, T^{S}, f^{S}\right)$, where $\Omega$ plays a role as $K$ in Theorem 3.1.

$\left(a_{1}\right)$ For each $x \in \Omega$ we have $T(x)=T^{S}(x) \neq \emptyset$. Hence condition (i) of Theorem 3.1 is valid.

$\left(a_{2}\right)$ For each $z \in \Omega$, we have

$$
\begin{aligned}
& \left\{x \in \Omega \mid \sup _{y \in T(x)}\left\langle u^{*}, f(x, y, z)\right\rangle \geq 0\right\} \\
= & \left\{x \in K \mid \sup _{y \in T(x)}\left\langle u^{*}, f(x, y, z)\right\rangle \geq 0\right\} \cap S .
\end{aligned}
$$

Since $S$ is finite-dimensional, the set

$$
\left\{x \in \Omega \mid \sup _{y \in T(x)}\left\langle u^{*}, f(x, y, z)\right\rangle \geq 0\right\}
$$

is closed. This implies that condition (ii) of Theorem 3.1 is valid.

$\left(a_{3}\right)$ Conditions (iii)-(vi) are automatically fulfilled.

By Theorem 3.1, there exists $x_{S} \in \Omega$ such that

$$
\max _{y \in T^{S}\left(x_{S}\right)}\left\langle u^{*}, f^{S}\left(x_{S}, \hat{y}, z\right)\right\rangle \geq 0, \quad \forall z \in \Omega .
$$

Since $T^{S}\left(x_{S}\right)=T\left(x_{S}\right), f^{S}\left(x_{S}, \hat{y}, z\right)=f\left(x_{S}, \hat{y}, z\right)$ we have

$$
\max _{y \in T\left(x_{S}\right)}\left\langle u^{*}, f\left(x_{S}, \hat{y}, z\right)\right\rangle \geq 0, \quad \forall z \in K \cap S .
$$

Put $Q_{S}=\{x \in K \mid x$ satisfies $(10)\}$. Then $Q_{S} \neq \emptyset$ because $x_{S} \in Q_{S}$. Moreover, the family $\left\{\bar{Q}_{S}: S \in \mathcal{F}\right\}$ has the finite intersection property. Indeed, taking any $V_{1}, V_{2} \in \mathcal{F}$ and putting $M=\operatorname{span}\left\{V_{1}, V_{2}\right\}$, we have

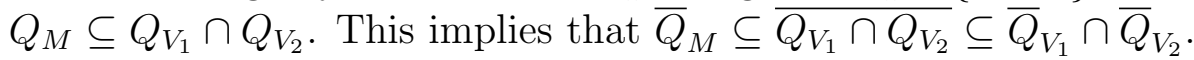

By condition (vi), $\bar{Q}_{S} \subset K_{2}$, which is compact. Then one has $\bigcap_{S \in \mathcal{F}} \bar{Q}_{S} \neq \emptyset$. Thus there exists $\hat{x} \in K_{2}$ such that $\hat{x} \in \bar{Q}_{S}$ for all 
$S \in \mathcal{F}$. Fix any $S \in \mathcal{F}$. Then there exists a net $\left\{x_{\alpha}\right\}_{\alpha \in \Lambda}$ in $Q_{S}$ such that $x_{\alpha} \rightarrow \hat{x}$. By the definition of $Q_{S}$ one has

$$
\max _{y \in T\left(x_{\alpha}\right)}\left\langle u^{*}, f\left(x_{\alpha}, y, z\right)\right\rangle \geq 0, \quad \forall z \in K \cap S .
$$

Fixing $z \in K \cap S$ and using assumption (ii), we obtain from (11) that

$$
\max _{\hat{y} \in T(\hat{x})}\left\langle u^{*}, f(\hat{x}, \hat{y}, z)\right\rangle \geq 0
$$

In summary, we have proved that

$$
\exists \hat{x} \in K_{2}, \forall z \in K \cap S, \quad \max _{y \in T(\hat{x})}\left\langle u^{*}, f(\hat{x}, y, z)\right\rangle \geq 0 .
$$

We now take any $v \in K$ and put $S^{\prime}=\operatorname{span}\{S, v\}$. Since $\hat{x}$ also satisfies (12) for $S^{\prime}$ we have

$$
\exists \hat{x} \in K_{2}, \quad \max _{y \in T(\hat{x})}\left\langle u^{*}, f(\hat{x}, \hat{y}, v)\right\rangle \geq 0 .
$$

Thus we have

$$
\exists \hat{x} \in K_{2}, \forall v \in K, \quad \max _{y \in T(\hat{x})}\left\langle u^{*}, f(\hat{x}, \hat{y}, v)\right\rangle \geq 0 .
$$

Using a similar argument as in the proof of Theorem 3.1, we show that there exists $\hat{y} \in T(\hat{x})$ such that $f(\hat{x}, \hat{y}, z) \nless 0$ for all $z \in K$. The proof is complete.

Theorem 3.5. Let $X, Y$ be locally convex spaces. In addition to conditions (i), (ii), (iii), (iv), (v) and (vi), suppose further that $K_{0}$ is weakly compact and $F(z)$ is weakly closed for all $z$ in $K$. Then there exists a point $\hat{x} \in K_{0}$ and $\hat{y} \in T(\hat{x})$ such that

$$
f(\hat{x}, \hat{y}, z) \nless 0, \quad \forall z \in K .
$$

Proof. For each absolutely convex weakly compact subset $S$ of $X$, let $X_{S}$ be the subspace of $X$ generated by $S$. Namely,

$$
X_{S}=\bigcup_{t>0} t S
$$

Equip $X_{S}$ with the norm defined by the gauge of $S$; namely,

$$
\|x\|=\inf \{t>0: x \in t S\} \text {. }
$$

Then $\left(X_{S},\|\cdot\|\right)$ is a dual Banach space by the Dixmier-Ng Theorem, see (Dixmier, 1948) and $(\mathrm{Ng}, 1972)$. Indeed, the predual of $\left(X_{S},\|\cdot\|\right)$ consists of those linear functionals on $X$ continuous on $S$. Hence, the weak* topology of $\left(X_{S},\|\cdot\|\right)$ is finer than the weak topology $\sigma\left(X_{S}, X^{\prime}\right)$ of $X_{S}$ inherited from $X$. As a result, $F(z)$ is weakly* closed in $\left(X_{S},\|\cdot\|\right)$ for all $z$ in $K \cap X_{S}$. 
Let $\mathcal{F}$ be the set of all dual Banach spaces $\left(X_{S},\|\cdot\|\right)$, where $S$ is an absolutely convex weakly compact subset of $X$ containing $K_{0}$. We can then proceed as in the proof of Theorem 3.4.

We remark that in Theorem 3.5 we can assume instead that $X$ is a topological vector space such that $X^{\prime}$ separates points in $X$. This condition is enough to ensure the conclusion of the Dixmier-Ng Theorem, as one can check by going through the proof of $\mathrm{Ng}$ (1972) carefully.

\section{Existence OF SOLUTION IN CASE OF STAR-PSEUdOMONOTONE OPERATORS}

We first introduce some notations on the star-pseudomonotone operator.

Definition 4.1. (a) The operator $T: K \rightarrow 2^{D}$ is called starpseudomonotone with respect to $f$ and $u^{*} \in C_{+}^{*}$ if for any $\left(x_{1}, y_{1}\right),\left(x_{2}, y_{2}\right) \in \Gamma_{T}$,

$\left\langle u^{*}, f\left(x_{1}, y_{1}, x_{2}\right)\right\rangle \geq 0 \quad$ implies $\quad\left\langle u^{*}, f\left(x_{1}, y_{2}, x_{2}\right)\right\rangle \geq 0$.

(b) $T$ is called star-quasimonotone with respect to $f$ and $u^{*} \in C_{+}^{*}$ if for any $\left(x_{1}, y_{1}\right),\left(x_{2}, y_{2}\right) \in \Gamma_{T}$,

$$
\left\langle u^{*}, f\left(x_{1}, y_{1}, x_{2}\right)\right\rangle>0 \quad \text { implies } \quad\left\langle u^{*}, f\left(x_{1}, y_{2}, x_{2}\right)\right\rangle \geq 0 .
$$

(c) $T$ is called star-properly quasimonotone with respect to $f$ and $u^{*} \in C_{+}^{*}$ if for any finite set $\left\{x_{1}, x_{2}, \ldots, x_{2}\right\}$ in $K$ and $x \in$ $\operatorname{co}\left\{x_{1}, x_{2}, \ldots, x_{2}\right\}$, there exists some $i \in\{1,2, \ldots, n\}$ such that

$$
\left\langle u^{*}, f\left(x, y, x_{i}\right)\right\rangle \geq 0, \quad \forall y \in T\left(x_{i}\right) .
$$

The notion of quasimonotone operators and pseudomonotone operators were first introduced by Karamardian and Schible (1990). For this case we put $Y=D=X^{*}, Z=\mathbb{R}, C=\mathbb{R}+, f(x, y, z)=\langle y, z-x\rangle$ and $u^{*}=1$. Then $T$ is a pseudomonotone operator (resp., quasimonotone) in the sense of theirs. While the notion of proper quasimonotonicity was introduced by Daniilidis and Hadjisavvas (1999). Also, putting $f(x, y, z)=\phi(y, z)-\phi(y, x), Z=\mathbb{R}, C=\mathbb{R}+$ and $u^{*}=1$ we obtain the notions of $\phi$-quasimonotone operators and $\phi$-pseudomonotone operators which have introduced recently by Aussel and Luc (2005).

The following are some relations among star-monotone operators.

Proposition 4.2. (a) If $T$ is star-pseudomonotone with respect to $f$ and $u^{*}$ then $T$ is also star-quasimonotone with respect to $f$ and $u^{*}$.

(b) Suppose $T$ is star-pseudomonotone with respect to $f$ and $u^{*}$, and the following conditions are fulfilled: 
(i) for each $x \in K$ and each $y \in T(x), f(x, y, x)=0$;

(ii) for each $x \in K$ and for each $y \in T(x)$, the function $f(x, y, \cdot)$ is $C$-convex.

Then $T$ is also star-properly quasimonotone with respect to $f$ and $u^{*}$.

Proof. (a) is obvious. For (b), let $\left\{x_{1}, x_{2}, \ldots, x_{n}\right\} \subset K$ and $x \in$ $\operatorname{co}\left\{x_{1}, x_{2}, \ldots, x_{n}\right\}$. Then $x=\sum_{i=1}^{n} t_{i} x_{i}$ with $t_{i} \geq 0$ and $\sum_{i=1}^{n} t_{i}=1$. By (i) we have $\left\langle u^{*}, f(x, y, x)\right\rangle=0$ for all $y \in T(x)$. Since $f(x, y, \cdot)$ is $C$-convex, by Proposition 2.2 the function $\left\langle u^{*}, f(x, y, \cdot)\right\rangle$ is convex. Hence

$$
0=\left\langle u^{*}, f(x, y, x)\right\rangle \leq \sum_{i=1}^{n} t_{i}\left\langle u^{*}, f\left(x, y, x_{i}\right)\right\rangle .
$$

It follows that there exists $i \in\{1,2, \ldots, n\}$ satisfying $\left\langle u^{*}, f\left(x, y, x_{i}\right)\right\rangle \geq 0$ for some $y \in T(x)$. Since $T$ is star-pseudomonotone, $\left\langle u^{*}, f\left(x, y, x_{i}\right)\right\rangle \geq$ 0 for all $y \in T\left(x_{i}\right)$. The proof is complete.

Definition 4.3. $T$ is called upper sign-continuous on $K$ with respect to $f$ and $u^{*} \in C_{+}^{*}$ if for all $x, z$ in $K$ the following is satisfied:

$$
\left(\forall t \in(0,1), \inf _{y \in T\left(x_{t}\right)}\left\langle u^{*}, f(x, y, z)\right\rangle \geq 0\right) \Longrightarrow \sup _{y \in T(x)}\left\langle u^{*}, f(x, y, z)\right\rangle \geq 0,
$$

where $x_{t}=(1-t) x+t z$.

The notion of upper sign-continuous operators was first introduced by Hadjisavvas (2003). In the above definition by putting $Y=D=X^{*}$, $Z=\mathbb{R}, C=\mathbb{R}_{+}, u^{*}=1$ and $f(x, y, z)=\langle y, z-x\rangle$ we also obtain the upper sign-continuous concept in the sense of Hadjisavvas.

The following lemma is a type of Minty's lemma.

Lemma 4.4. Suppose that $Y$ is locally convex. Let $u^{*} \in C_{+}^{*}, \hat{x} \in K$ and the following conditions hold:

(i) for each $x \in K$ and $y \in D$ one has $f(x, y, x)=0$;

(ii) for each $x \in K$ and $y \in T(x)$ the function $f(x, y, \cdot)$ is $C$-convex and $C$-lower semicontinuous;

(iii) for each $(x, z) \in K \times K$, the function $f(x, \cdot, z)$ is $C$-concave and $C$-upper semicontinuous;

(iv) $T$ is upper sign-continuous with respect to $f$ and $u^{*}$, with weakly compact and convex values;

(v) $\left\langle u^{*}, f(\hat{x}, y, z)\right\rangle \geq 0$ for all $z \in K$ and $y \in T(z)$.

Then $\hat{x} \in S_{V}$. 
Proof. Rewrite condition (v) as

$$
\forall z \in K, \forall y \in T(z), \quad\left\langle u^{*}, f(\hat{x}, y, z)\right\rangle \geq 0 .
$$

Taking any $z \in K$, we have $z_{t}=(1-t) \hat{x}+t z \in K$ for $t \in(0,1)$. Hence (13) implies that

$$
\left\langle u^{*}, f\left(\hat{x}, y, z_{t}\right)\right\rangle \geq 0, \quad \forall y \in T\left(z_{t}\right) .
$$

We claim that

$$
\left\langle u^{*}, f(\hat{x}, y, z)\right\rangle \geq 0, \quad \forall y \in T\left(z_{t}\right) .
$$

Suppose, on contrary, that the assertion is false. Then there exists $t \in(0,1)$ and $y_{t} \in T\left(z_{t}\right)$ such that

$$
\left\langle u^{*}, f\left(\hat{x}, y_{t}, z\right)\right\rangle<0 .
$$

By (ii) and Proposition 2.2 we have

$$
t\left\langle u^{*}, f\left(\hat{x}, y_{t}, z\right)\right\rangle+(1-t)\left\langle u^{*}, f\left(\hat{x}, y_{t}, \hat{x}\right)\right\rangle \geq\left\langle u^{*}, f\left(\hat{x}, y_{t}, z_{t}\right)\right\rangle .
$$

Combining this and (i) we obtain

$$
t\left\langle u^{*}, f\left(\hat{x}, y_{t}, z\right)\right\rangle \geq\left\langle u^{*}, f\left(\hat{x}, y_{t}, z_{t}\right)\right\rangle .
$$

From here and (16), it follows that

$$
\left\langle u^{*}, f\left(\hat{x}, y_{t}, z_{t}\right)\right\rangle<0,
$$

which contradicts (14). Thus our claim is verified.

By (15) we obtain

$$
\inf _{y \in T\left(z_{t}\right)}\left\langle u^{*}, f(\hat{x}, y, z)\right\rangle \geq 0 .
$$

By the upper sign-continuity of $T$, we have

$$
\sup _{y \in T(\hat{x})}\left\langle u^{*}, f(\hat{x}, y, z)\right\rangle \geq 0 .
$$

This implies that

$$
\inf _{z \in K} \sup _{y \in T(\hat{x})}\left\langle u^{*}, f(\hat{x}, y, z)\right\rangle \geq 0 .
$$

Since the function $y \mapsto\left\langle u^{*}, f(\hat{x}, y, z)\right\rangle$ is upper semicontinuous and concave, it is also upper semicontinuous in the weak topology of $Y$. According to the minimax theorem (Theorem 2.4) we obtain from (21) that

$$
\max _{y \in T(\hat{x})} \inf _{z \in K}\left\langle u^{*}, f(\hat{x}, y, z)\right\rangle=\inf _{z \in K} \max _{y \in T(\hat{x})}\left\langle u^{*}, f(\hat{x}, y, z)\right\rangle \geq 0 .
$$

Hence there exists $\hat{y} \in T(\hat{x})$ such that

$$
\inf _{z \in K}\left\langle u^{*}, f(\hat{x}, \hat{y}, z)\right\rangle \geq 0 \text {. }
$$

This means that

$$
\left\langle u^{*}, f(\hat{x}, \hat{y}, z)\right\rangle \geq 0, \quad \forall z \in K
$$


By (5) we obtain

$$
f(\hat{x}, \hat{y}, z) \nless 0, \quad \forall z \in K .
$$

Hence $\hat{x} \in S_{V}$.

Based on Lemma 4.4, we have the following existence result of strong solutions for $\operatorname{GVVI}(K, T, f)$.

Theorem 4.5. Let $X$ be a reflexive Banach space. Let $D$ be a nonempty set in the locally convex space $Y, K$ be a nonempty convex set in $X$, and $K_{0}$ be a weakly compact subset of $K$. Let $T: K \rightarrow 2^{D}$ be a multifunction, $f: K \times D \times K \rightarrow Z$ be a single-valued mapping and $u^{*} \in C_{+}^{*}$ be given. Assume that:

(a) for each $x \in K$ and $y \in D, f(x, y, x)=0$;

(b) for each $x \in K$ and for each $y \in T(x)$, the function $f(x, y, \cdot)$ is $C$-strongly convex and lower semicontinuous;

(c) for each $z \in K$ the function $f(\cdot, \cdot, z)$ is $C$-concave and upper semicontinuous;

(d) $T$ is star-pseudomonotone and upper sign-continuous with respect to $f$ and $u^{*}$;

(e) for each $x \in K, T(x)$ is a weakly compact and convex set;

(f) for each $x \in K \backslash K_{0}$ there exists $z \in K_{0}$ such that $f(x, y, z)<0$ for all $y \in T(x)$.

Then there exist $\hat{x} \in K_{0}$ and $\hat{y} \in T(\hat{x})$ such that $f(\hat{x}, \hat{y}, z) \nless 0$ for all $z \in K$.

Proof. We choose $r>0$ such that $K_{0} \subset \operatorname{int} B_{r}$ where $B_{r} \subset X$ is the closed ball with radius $r$. Put $\Omega=K \cap B_{r}$. We next define the multifunction $G: \Omega \rightarrow 2^{X}$ by setting

$$
G(z)=\left\{x \in \Omega \mid\left\langle u^{*}, f(x, y, z)\right\rangle \geq 0, \forall y \in T(z)\right\} .
$$

By Proposition 4.2, $T$ is also star-properly quasimonotone with respect to $f$ and $u^{*}$. Hence for any finite subset $\left\{z_{1}, z_{2}, \ldots, z_{n}\right\}$ in $\Omega$ and for every $z \in \operatorname{co}\left\{z_{1}, z_{2}, \ldots, z_{n}\right\}$ there exists $i \in\{1,2, . ., n\}$ such that

$$
\left\langle u^{*}, f\left(z, y, z_{i}\right)\right\rangle \geq 0, \quad \forall y \in T\left(z_{i}\right) .
$$

This implies that $z \in G\left(z_{i}\right)$ and so

$$
\operatorname{co}\left\{z_{1}, z_{2}, \ldots, z_{n}\right\} \subseteq \bigcup_{i=1}^{n} G\left(z_{i}\right) .
$$

By (c) and Proposition 2.2, the mapping $\left\langle u^{*}, f(\cdot, y, z)\right\rangle$ is concave and u.s.c. on $\Omega$. Hence $G(z)$ is a closed convex set. Consequently, $G(z)$ is a weakly compact subset of $\Omega$. Thus $G$ is a KKM mapping. By Theorem 
2.3 , there exists $\hat{x} \in \Omega$ such that $\hat{x} \in G(z)$ for all $z \in \Omega$. This implies that

$$
\left\langle u^{*}, f(\hat{x}, y, z)\right\rangle \geq 0, \quad \forall z \in \Omega, \quad \forall y \in T(z) .
$$

By Lemma $4.4, \hat{x}$ is a strong solution of $\operatorname{GVVI}(\Omega, T, f)$. This means that

$$
\exists \hat{y} \in T(\hat{x}), \forall z \in \Omega, \quad f(\hat{x}, y, z) \nless 0 .
$$

Combining this and (f) we get $\hat{x} \in K_{0} \subset \operatorname{int} B_{r}$.

It remains to show that

$$
\exists \hat{y} \in T(\hat{x}), \forall z \in K \quad f(\hat{x}, y, z) \nless 0 .
$$

Taking any $z \in K$ we have $z_{t}=\hat{x}+t(z-\hat{x}) \in \Omega$ for a sufficiently small $t \in(0,1)$. Hence (18) implies that

$$
f\left(\hat{x}, \hat{y}, z_{t}\right) \nless 0 .
$$

If $f(\hat{x}, \hat{y}, z)<0$ then by (b) we have

$$
t f(\hat{x}, \hat{y}, z)+(1-t) f(\hat{x}, \hat{y}, \hat{x})-f\left(\hat{x}, \hat{y}, z_{t}\right) \in \operatorname{int} C \cup\{0\} .
$$

Hence

$$
\begin{aligned}
-f\left(\hat{x}, \hat{y}, z_{t}\right) & \in-t f(\hat{x}, \hat{y}, z)+\operatorname{int} C \cup\{0\} . \\
& \subset \operatorname{tint} C+\operatorname{int} C \cup\{0\} . \\
& \subset \operatorname{int} C+\operatorname{int} C \cup\{0\} . \\
& =\operatorname{int} C .
\end{aligned}
$$

This implies that $f\left(\hat{x}, \hat{y}, z_{t}\right)<0$, which contradicts $(20)$. Thus we must have $f(\hat{x}, \hat{y}, z) \nless 0$ and so (19) is fulfilled. The proof is complete.

\section{REFERENCES}

[1] Aubin, J.P. (1979), Mathematical Methods of Game and Economic Theory, Amsteredam.

[2] Ansari, Q.H. and Yao, J.C. (1999a) An existence result for the generalized vector equilibrium problem, Applied Mathematics Letters, 12, 53-56.

[3] Ansari, Q.H. and Yao, J.C. (1999b) On strong solution of the the generalized implicit vector variational inequality problem, Advanced Nonlinear Variational Inequalities 2, 1-10.

[4] Aussel, D. and Hadjisavvas, N. (2004) On quasimonotone variational inequalities, Journal of Optimzation Theory and Applications 121, 445-450.

[5] Aussel, D. and Luc, D. T. (2005) Existence conditions in general quasimonotone variational inequlities, Bulletin of Australian Mathematical Society (to appear).

[6] Bianchi, M., Hadjisavvas, N. and Shaible, S. (1997), Vector equilibrium problems with generalzed Monotone bifunctions, Journal of Optimzation Theory and Applications 92, 527-452. 
[7] Blum, E. and Oettli, W. (1996), From optimization and variational inequalities to equilibrium problems, The Mathematics Student 63, 123-145.

[8] Chan, D. and Pang, J.S. (1982), The generalized quasi-variational inequality problem, Mathematics of Operations Research 7, 211-222.

[9] Chen. G.Y. and Craven, B.D. (1990), A vector variational inequality and optimization over an efficient set, ZOR-Meth, Models of Operation Research 3, $1-12$.

[10] Cubiotti, P. and Yen, N.D. (1997), A results related to Ricceri's conjecture on generalized quasivariational inequalities, Archiv der Mathematik 69, 507-514.

[11] Cubiotti, P. (2002), On the discontinuous infinite-dimensional generalized quasivariational inequality problem, Journal of Optimization Theory and Applications 115, 97-111.

[12] Cubiotti, P. (2003), Existence theorem for the discontinuous generalized quasivariational inequality problem, Journal of Optimization Theory and Applications 119, 623-633.

[13] Cubiotti, P. and Yao, J.C. (1997), Discontinuous implicit quasi-variational inequalities with applications to fuzzy mappings, Mathematical Methods of Operations Research 46, 213-328.

[14] Cubiotti, P. and Yao, J.C. (2005), Discontinuous implicit generalized quasivariational inequalities in Banach spaces, submitted for publication.

[15] Daniilidis, A. and Hadjisavvas, N. (1999), Characterization of nonsmooth semistrictly quasiconvex and strictly quasiconvex functions, Journal of $\mathrm{Op}$ timization Theory and Applications 102, no. 3, 525-536.

[16] Dixmier, J. (1948), Sur un theoreme de Banach, Duke Math. J. 15, 1057-1071.

[17] Hadjisavvas, N. (2003), Continuity and maximality properties of pseudomonotone operators, Journal of Convex Analysis 10, 465-475.

[18] Hou, S.H., Yu, H. and Chen, G.Y. (2003), On vector quasi-equilibrium problems with set-valued maps, Journal of Optimization Theory and Applications $119,485-498$.

[19] Jeyakumar, V., Oettli, W. and Natividad, M. (1993), A solvability Theorem for a Class of Quasiconvex mappings with Applications to Optimization, Journal of Mathematical Analysis and Applications. 179, 537-546.

[20] Karamardian, S. and Shaible, S. (1990), Seven kinds of monotone maps, Journal of Optimization Theory and Applications 66, 37-46.

[21] Kien, B.T., Wong, N. C. and Yao, J.C.(2005), On the solution existence of generalized quasivariational inequalities with discontinuous mappings, submitted for publication.

[22] Kien, B.T., Wong, N. C. and Yao, J.C.(2005): On the solution existence of implicit generalized quasivariational inequalities with discontinuous mappings, submitted for publication.

[23] Lee, B.S., Lee, G.M. and Kim, D.S. (1997) Generalized vector variational-like inequalities in locally convex topological vector spaces, Indian Journal of Pure and Applied Mathematics 28, 33-41.

[24] Li, J. (2004), On the existence of solution of variational inequalities in Banach spaces, Journal of Mathematical Analysis and Applications 295, 115-126.

[25] Luc, D.T.(1989), Theory of Vector Optimization, Springer, Berlin.

[26] Ng, K. F. (1972), On a theorem of Dixmier, Math. Scand. 29, 279-280.

[27] Oettli, W.(1997), A Remark on vector-valued equilibria and generalized monotonicity, Acta Mathematica Vietnamica 22, 213-221. 
[28] Schaible, S. and Yao, J.C. (1995), On the equivalence of nonlinear complementarity problem and least element problems, Mathematical Programming 70, 191-200.

[29] Tanaka, T. (1997), Generalized semicontiuity and Existence theorems for Cone saddle point, Applied Mathematics and Optimization 36, 313-322.

[30] Yao, J.C. and Guo, J.S. (1994), Variational and generalized variational inequalitieties with discontinuous mappings, Journal of Mathematical Analysis and Applications 182, 371-382.

[31] Yao, J.C. (1995), Generalized quasivariational Inequalitiety problems with discontinuous mappings, Mathematics of Operations Research 20, 465-478.

[32] Yao, J.C. and Guo, J.S. (1992), Extension of strongly nonlinear quasivariational inequalities, Applied Mathematics Letters 5, 35-38.

[33] Yao, J.C. (1994), Variational Inequalities with Generalized Monotone Operators, Mathematics of Operations Research 19, 691-705.

[34] Yen, N.D.(1995), On an existence theorem for generalized quasivariational inequalities, Set-Valued Analysis 3, 1-10.

[35] Yen, N.D., On a class of discontinuous vector-valued functions and the associated quasivariational inequalities, Optimization. 30 (1994), 197-202.

[36] Zeidler, E.(1993), Nonlinear Functional Analysis and Its Application, I FixedPoint Theorems, Springer-Verlag.

[37] Zeidler, E. (1990), Nonlinear Functional Analysis and Its Application, II/B: Nonlinear Monotone Operators, Springer-Verlag. 